\title{
Geotecnologias na gestão de reservatórios: uma revisão e uma proposta de integração
}

\author{
Geotechnologies on water reservoir management: \\ a review and an integration proposal
}

Maria do Carmo Sobral', Helio Lopes², Ana Lúcia Candeias³, Gustavo Melo4, Günter Gunkel

\begin{abstract}
RESUMO
Este trabalho teve como foco apresentar uma visão geral dos sistemas de informações geográficas (SIGS) e do sensoriamento remoto (SR), com ênfase nas suas aplicações para obtenção de informações da massa d'água. Essas informações são integradas ao Quadro da Diretiva da Água, e nesse sentido, busca-se propor a ligação dessas informações à Resolução no 357/2005, de forma a servir de ferramenta para a tomada de decisão em uma gestão estratégica da qualidade da água, principalmente em grandes reservatórios. Assim, é realizada uma revisão integrando as geotecnologias à ciência limnológica e à gestão de reservatórios, ressaltando os possíveis potenciais da interação interdisciplinar. Por fim, é esquematizado um modelo integrando SIGs, SR e limnologia para o monitoramento e a gestão dos padrões de qualidade da água conforme a Resolução no 357/2005.
\end{abstract}

Palavras-chave: qualidade da água; geotecnologias; legislação hídrica.

\begin{abstract}
An overview of geographical information system (GIS) and remote sensing (RS) is the main focus of this work, that emphasizes their application to obtain water body information. This information is integrated to the Framework Directive, and in this way, seeks to propose the linkage of these information to the Resolution 357/2005, in order to serve as a tool for decision making in strategic management of water quality mainly in large reservoirs. Thus, a review that integrates geotechnology to limnological science and to reservoirs management was done, highlighting the possible potential of interdisciplinary integration. At the end, an integration model of GIS, RS and limnology is schematized for monitoring and management of water quality standards according to the Resolution 357/2005.
\end{abstract}

Keywords: water quality; geotechnology; water legislation.

\section{INTRODUÇÃO}

Nas últimas décadas, vem se intensificando cada vez mais a poluição dos solos e, consequentemente, dos sistemas aquáticos, em decorrência principalmente do desmatamento e do uso intensivo do solo. Tais fenômenos causam erosão com carreamento de fertilizantes para os corpos hídricos (reservatórios), aumentando diversos constituintes na água.

Conforme Melo (2011), a tipologia e a caracterização de um reservatório são fatores importantes para a implantação de planejamento e gestão. O conhecimento sobre o sistema de operação de um reservatório e as informações armazenadas nesses ecossistemas artificiais tornam-se extremamente relevantes quando se considera o seu uso potencial para o planejamento e gerenciamento de uma bacia hidrográfica, incluindo o controle da qualidade da água.
Normas ambientais têm sido regulamentadas buscando o controle da qualidade da água em diversos reservatórios em todo o mundo. Nos Grandes Lagos da América do Norte, a concentração de nitrato nas águas continua aumentando em muitas bacias hidrográficas. Espécies de fitoplânctons no Lago Erie mostram transição de espécies associadas com condições de eutrofização, necessitando de um monitoramento para avaliação do corpo hídrico (FUKUE; SATO; MULLIGAN, 2011).

Muitas tecnologias existentes para o monitoramento de algas e cianobactérias são técnicas microscópicas laboriosas e altamente variáveis. $\mathrm{O}$ espectro eletromagnético utilizado em sensoriamento remoto (SR) vem sendo aplicado por meio de instrumentação algal, denominado fluorômetro, para monitoramento da coluna d'água (GREGOR \& MARSÁLEK, 2004). O sensor pode detectar clorofila no primeiro

$\square$

Professora do Departamento de Engenharia Civil da Universidade Federal de Pernambuco (UFPE) - Recife (PE), Brasil.

2Doutorando do Programa de Pós-Graduação em Engenharia Civil da UFPE (falecido) - Recife (PE), Brasil.

${ }^{3}$ Professora do Departamento de Engenharia Cartográfica da UFPE - Recife (PE), Brasil.

${ }^{4}$ Doutor em Engenharia Civil pela UFPE - Recife (PE), Brasil.

${ }^{5}$ Professor do Departamento de Engenharia Ambiental, Centro de Limnologia, Universidade Técnica de Berlim - Berlim, Alemanha.

Endereço para correspondência: Maria do Carmo Sobral - Avenida Acadêmico Hélio Ramos, s/n - Cidade Universitária - 50740-530 - Recife (PE), Brasil - E-mail: msobral@ufpe.br Recebido: 06/02/13 - Aceito: 29/08/16 - Reg. ABES: 111054 
canal (440 e $680 \mathrm{~nm}$ ) e ficocianina de cianobactérias no segundo (620 e $645 \mathrm{~nm}$ ), podendo ser usado para monitoramento pontual em tempo real na coluna d'água.

Zhang et al. (2011) afirmam que a aplicação dessas técnicas pode ajudar pesquisadores, gestores ambientais e tomadores de decisão a conhecerem quando, onde e por que a proliferação demasiada de algas ocorre em determinado corpo hídrico. Dessa forma, para se ter um melhor entendimento, técnicas de SR podem ser usadas suplementarmente às observações de campo e aos experimentos de laboratório.

Autores como Le et al. (2009), Ma e Dai (2005) e Zhang et al. (2011) conduziram uma análise para obter a distribuição temporal e espacial de clorofila-a e a proliferação de algas no Lago Taihu, China.

Goddijn-Murphy et al. (2009) observam que o SR oferece diversas técnicas para o monitoramento de recursos hídricos em diferentes áreas e escalas de tempo. Além disso, existe também uma variabilidade de parâmetros a serem monitorados por SR: transparência secchi (KLOIBER; BREZONIK; BAUER, 2002), clorofila-a (CHEN et al., 2011; GITELSON et al., 2008; KLOIBER; BREZONIK; BAUER, 2002; ZHANG et al., 2011), carbono orgânico particulado (HADJIMITSIS \& CLAYTON, 2011), sólidos suspensos totais (ARTIGAS et al., 2008; WANG et al., 2006), turbidez (WANG et al., 2006), entre outros.

O monitoramento em escala regional é fundamental na geração de informações para decisões na gestão de lagos (KLOIBER; BREZONIK; BAUER, 2002). Dados de programas de monitoramento são frequentemente usados para estimar variações na qualidade da água em corpos hídricos de uma região, examinar diferenças regionais e investigar relações entre as condições da paisagem (uso e cobertura do solo) e da qualidade da água. Essas informações podem ser usadas para desenvolver critérios da qualidade da água e guiar decisões referentes ao manejo (KLOIBER; BREZONIK; BAUER, 2002). Uma correta arquitetura estrutural de programa de monitoramento deve ter uma adequada representação no espaço e no tempo para prover informações necessárias às propostas científicas e de gestão. No entanto, o arranjo da escala regional de programas de monitoramento frequentemente requer um equilíbrio entre detalhes espaciais e temporais, mas devido a problemas logísticos e de custo, a cobertura espacial é sacrificada em favor de maior frequência amostral (KLOIBER; BREZONIK; BAUER, 2002). Para cobrir esse vazio, o SR pode fornecer algumas características do corpo hídrico, gerando informações com certo nível de precisão, pois os dados espectrais gerados pelo sensor são correlacionados a diversas variáveis de qualidade da água que afetam as propriedades ópticas do corpo hídrico em estudo (HADJIMITSIS \& CLAYTON, 2011).

Os dados coletados espacial e temporalmente por SR podem ser integrados a uma política de monitoramento de recursos hídricos, conforme limiar estabelecido em lei de recursos hídricos. Pode ser observado que, na Diretiva Quadro da Água (DQA) nº 2000/60/CE, da União Europeia, o monitoramento por satélite já vem sendo sugerido e implementado para se obter informações sobre a qualidade dos corpos hídricos, porém ainda há incertezas que necessitam ser modeladas para se obter uma melhor otimização dos algoritmos e, consequentemente, uma melhor precisão de acordo com os limiares estabelecidos na política de recursos hídricos.

Nesse sentido, este trabalho objetivou revisar a interligação de parâmetros de qualidade da água com modelagem por técnicas de SR guiados por limiares para corpos hídricos estabelecidos na Resolução no 357/2005 (BRASIL, 2005)

Um monitoramento que explore as vertentes espacial e temporal é fundamental para se entender a dinâmica desse processo de modo a promover subsídios para a tomada de decisão em escala de reservatório. Dessa forma, os sistemas de informação geográfica (SIGs) e o SR são ferramentas que contemplam esses pré-requisitos para a geração de informação sobre ecossistemas aquáticos de grande porte.

\section{PROCESSAMENTO DE DADOS ESPACIAIS NA GESTÃO DE RESERVATÓRIOS}

Diversas tecnologias de coleta de informações espaciais estão sendo usadas para entender o comportamento limnológico de reservatórios, lagos e rios (ALCÂNTARA; NOVO; STECH, 2011). As principais ferramentas utilizadas para análise espacial de dados limnológicos de reservatórios são o SR e os SIGs, trazendo subsídios para a tomada de decisão e, por conseguinte, auxiliando em um plano de gestão ambiental de um determinado corpo hídrico (BABAN, 1999; NELLIS; HARRINGTON; WU, 1998; CHEN; ZHANG; HALLIKAINEN, 2007). Essas áreas do conhecimento são fundamentais no processamento e na geração de informações em plantas, mapas e relatórios, e conforme Sánchez (2008) são essenciais para prover e sintetizar informações em qualquer estudo ambiental.

As maiores fontes de degradação de recursos hídricos superficiais e subterrâneos têm origem na poluição agrícola, industrial e de áreas urbanas (ALEXANDRIDIS et al., 2007; ATKINSON \& CANTER, 2011). O plano de recuperação da qualidade da água é dificultado pela falta de dados suficientes à modelagem e à geração de informações (SÁNCHEZ, 2008), com lacunas evidentes nas dimensões espaciais e temporais (ALEXANDRIDIS et al., 2007). Em estudos realizados no Lago Korônia, na Grécia, Alexandridis et al. (2007) empregaram técnicas de geoprocessamento, SR, processamento digital de imagens e visualização geográfica em camadas, com o objetivo de suprir lacunas usando processamento de imagens, dados hidrológicos e hidrogeológicos, para a construção do plano de restauração. O método empregado por Alexandritis et al. (2007), aplicando geotecnologias, teve como objetivo modelar um cenário sustentável para o Lago Korônia. Para isso, dados atuais e passados de SR foram usados para avaliar o estado atual e o nível de degradação, de forma a definir melhor o meio de restauração. 
Diversas camadas de informação geográfica podem ser geradas usando dados de SR em um ambiente de SIGs. Segundo Alexandridis et al. (2007), essas informações, aliadas a dados de campo, são usadas para conhecer melhor o estado atual e o nível de degradação de um determinado local geográfico. Por conseguinte, podem prover dados ambientais uteis à tomada de decisão.

O SR tem sido utilizado para monitoramento de parâmetros da superfície terrestre, incluindo:

- mudança de uso e cobertura do solo (LOPES et al., 2010; SILVEIRA \& SILVA, 2010; ZHANG et al., 2009; ZHANG; ZHENGJUN; XIAOXIA, 2009);

- balanço de energia do solo (RODRIGUES et al., 2009);

- estimativas de evapotranspiração (TUCCI \& MENDES, 2006; OLIVEIRA et al., 2014);

- estimativas de biomassa (WANG; SHI; TANG, 2011) em bacias hidrográficas (POWELL et al., 2010);

- mapeamento de carbono no solo (MEERSMANS et al., 2008; ANDERSON et al., 2008; FUCHS et al., 2009).

Também tem sido empregado em ecossistemas aquáticos para:

- estimativa de temperatura (ALCÂNTARA; NOVO; STECH, 2011; GIARDINO et al., 2001; KAY et al., 2005; MATSUOKA et al., 2011, MATOS et al., 2015);

- avaliação da qualidade da água, espacializando o índice de estado trófico e monitoramento da eutrofização (CHENG \& LEI, 2001; DUAN et al., 2008; SASS et al., 2007; ZHENGJUN; JIANMING; GUISEN, 2008, LOPES et al., 2015);

- avaliação da produção primária em reservatórios (BERGAMINO et al., 2010).

Impactos antropogênicos também têm sido estudados com técnicas de SR e SIGs, incluindo fontes pontuais e difusas (JOHNSON, 2009; TRIPATHI et al., 1998).

Uma análise funcional voltada à bacia hidrográfica é necessária para desenvolver um plano de restauração sustentável (ALEXANDRIDIS et al., 2007). Na gestão hidroecológica de uma bacia, há a necessidade de informações espaciais e temporais que constituam subsídio à tomada de decisão em diversos planos de manejo hidroambiental. Conforme Zalidis et al. (2002), um plano de restauração procura identificar quais processos estão impactados e quais funções hidroecológicas necessitam ser restauradas. Dessa forma, um primeiro passo para a restauração de um corpo hídrico é a avaliação da bacia hidrográfica de forma a identificar as primeiras forças de degradação, bem como os resultados da pressão no ecossistema aquático (ALEXANDRIDIS et al., 2007).

Nesta avaliação, dados geográficos históricos relacionados ao estado do ecossistema, derivados de análise de imagens de satélite, são empregados em SIGs para examinar o processo de degradação. Conforme Johnson (2009), um exemplo de uma nova tecnologia de mapeamento é a imagem de satélite, a partir da qual mapas detalhados da superfície terrestre podem ser criados. Segundo Johnson (2009), parâmetros de qualidade da água (como sedimentos suspensos, algas, matéria orgânica dissolvida, óleos, plantas aquáticas, variação termal) mudam o espectro de energia solar refletida e/ou da radiação termal emitida da superfície da água, e essa radiação pode ser medida usando técnicas de SR.

Planos de bacias hidrográficas e modelos de gestão podem ser integrados a um SIG. Modelos para bacias hidrográficas incluem aqueles que simulam e otimizam a operação de reservatórios e instalações associadas para servir a múltiplas propostas. De acordo com Alexandridis et al. (2007), o SIG é usado, principalmente, para visualizar, analisar e combinar dados, de forma a gerar informações relacionadas ao estado ecológico de uma bacia, à disponibilidade de recursos naturais na área e à avaliação da pressão e dos impactos da atividade humana em sistemas aquáticos (lagos, reservatórios, rios).

Nas últimas décadas, pesquisadores de sistemas aquáticos têm ressaltado a importância e os benefícios de dados de SR para melhorar o conhecimento acerca do funcionamento desses ecossistemas (LEUVEN; POUDEVIGNE; TEEUW, 2002). Isso porque existe a necessidade de se entender o funcionamento do ecossistema levando-se em conta a heterogeneidade espacial e a variabilidade temporal. Baker, Young e Arocena (2000) estudaram o impacto da tempestade de poeira em reservatório e como tal fenômeno afeta comunidades; a pesquisa indicou a necessidade de se conhecer espacialmente a área de forma a estabelecer um programa de plantio para mitigar os efeitos dos fenômenos. Para o estabelecimento da gestão do reservatório, foram integrados aspectos sociais e físicos de forma a prover uma visão geral a partir dos planos de informação social, da análise de solos e do inventário florestal.

Dados de satélite vêm sendo utilizados, também, no auxílio a políticas de recursos hídricos e legislação. Conforme Paes, Candeias e Sobral (2010), o uso do SIG como ferramenta de suporte à tomada de decisão tem crescido exponencialmente nos últimos anos. O monitoramento da qualidade da água usando SR, com foco na sua distribuição espacial por meio de observações com imagens de satélites, fornece suporte a uma política de recursos hídricos. No seu estudo, Chen, Zhang e Hallikainen (2007) apresentam uma espacialização da concentração de nitratos de forma a auxiliar na tomada de decisão conforme a DQA $n^{\circ}$ 2000/60/CE. Melo (2011) realizou estudo de parâmetros de qualidade da água em reservatórios do nordeste, a fim de verificar temporalmente os valores que estão acima do estabelecido pela Resolução n 357/2005 (BRASIL, 2005). A implantação da diretiva de recursos hídricos e o alcance dos objetivos referente à qualidade da água têm grande dependência no conhecimento científico sobre o estado dos 
recursos hídricos e dos efeitos das medidas tomadas (CHEN; ZHANG; HALLIKAINEN, 2007).

Melo (2007) avaliou espacial e temporalmente os parâmetros de qualidade da água, tomando como base os limiares estabelecidos nas condições e nos padrões de qualidade das águas. Nesse sentido, no desenvolvimento deste item, objetivou-se organizar um esquema de aplicação do SIG e do SR em sistemas aquáticos, para gestão do corpo hídrico, tendo como área de estudo o reservatório de Itaparica e seu entorno, bem como integrar a Resolução no 357/2005 em conjunto com SIG e SR, de forma a verificar parâmetros relevantes em que tais sistemas possam auxiliar na sua determinação e no seu monitoramento.

\section{MONITORAMENTO DA QUALIDADE DA ÁGUA}

Conforme Jessel e Jacobs (2005), a implementação da DQA traz padrões de uso do solo em bacias hidrográficas para a redução de poluição difusa, visando alcançar um bom estado ecológico, ou um bom potencial ecológico nos corpos hídricos superficiais, referente à qualidade da água. Segundo Sobral et al. (2008), a DQA representa um laboratório das políticas da água no qual se pretende estabelecer, de forma efetiva, bases comuns para a gestão dos recursos hídricos em um espaço marcado por diversidade geográfica, socioeconômica e cultural.

No monitoramento da qualidade da água, satélites de captação de informações da superfície terrestre armazenam regularmente dados espaciais utilizados em algoritmos da qualidade da água. Essas informações modeladas e espacializadas sobre a qualidade da água praticamente seriam os dados de saída, ligados a uma tabela de classificação das águas para detectar e monitorar se estão dentro do limite ou excedem valores críticos de padrões de qualidade da água estabelecidos em resolução, no Brasil a Resolução no 357/2005.

Para essa interação relacionada ao monitoramento da qualidade da água, cinco passos são necessários, conforme Chen, Zhang e Hallikainen (2007):

1. identificação de medições apropriadas da qualidade da água e de indicadores do grau de degradação do corpo hídrico;

2. monitoramento da qualidade da água usando dados de SR;

3. distribuição espacial de informação da qualidade da água em relação ao ecossistema aquático e às mudanças ambientais;

4. ligação alfanumérica entre monitoramento da qualidade da água e políticas de recursos hídricos;

5. avaliação completa de monitoramento da qualidade da água usando SR na diretiva dos recursos hídricos.

No primeiro passo, técnicas de medidas e indicadores variam conforme a bacia hidrográfica e os corpos d'água, os quais são dependentes dos usos atuais, dos impactos e das características da paisagem, e são incorporados em um plano de gestão baseado em SIG. De acordo com Xingtang et al. (2004), a resposta a como proteger o meio ambiente e efetivamente prevenir e reduzir desastres é um ponto-chave no desenvolvimento regional sustentável, especialmente no uso de modernas e avançadas tecnologias de informações espaciais, incluindo SR, SIGs, sistema de posicionamento global e suas tecnologias integradas. Um plano baseado em SIG deve incluir: o impacto de atividades humanas nas águas superficiais; a análise econômica do uso da água; o programa de coletas; informações públicas e consultas; e a variação sazonal de todas essas medidas.

Em relação ao segundo passo, observações de satélites são usadas para prover a distribuição espacial de informações da qualidade da água, como clorofila-a, matéria suspensa total, substâncias amarelas, turbidez, profundidade secchi (transparência) e temperatura da superfície da água da área em estudo (CHEN; ZHANG; HALLIKAINEN, 2007; WANG et al., 2010). Conforme Xingtang et al. (2004), para o controle da poluição dos recursos hídricos, especialmente em bacias hidrográficas e lagos continentais, dados de SR, como TM, SPOT, MODIS, ASTER e HYPERION, são usados para capturar informações da área pesquisada quanto ao corpo hídrico e às medidas de superfície sobre a qualidade da água. Por exemplo, dados de clorofila-a são usados para analisar mudanças de indicadores importantes de qualidade da água em escala espacial (CHEN; ZHANG; HALLIKAINEN, 2007; ZHANG et al., 2009; LOPES et al., 2015). Por conseguinte, concentrações de nitrogênio e fósforo, devido à sua distribuição espacial no corpo d'água, podem ser correlacionadas.

Quanto ao terceiro passo, a análise é feita com base, principalmente, no grau de mudanças dos parâmetros biofísicos e/ou ambientes socioeconômicos, que podem impactar os processos hidrológicos, todo o balanço da água, e o uso sustentável da água na bacia hidrográfica em determinado período do ano. Segundo Sobral et al. (2008), os parâmetros relativos à natureza físico-química da água, às características hidrodinâmicas e à estrutura física dos meios hídricos fazem parte da definição dos diversos tipos de água. A distribuição espacial da qualidade da água está relacionada diretamente com o sistema aquático, as margens inundáveis e as mudanças no meio ambiente. Essas mudanças incluem mudança no uso e na cobertura do solo (por exemplo, desmatamento), mudanças demográficas, desenvolvimento industrial e formas do uso da água (CHEN; ZHANG; HALLIKAINEN, 2007; FUKUE; SATO; MULLIGAN, 2011).

No quarto passo, o banco de dados relacionado à classificação de parâmetros de qualidade da água pode estar conectado à distribuição espacial de informações de qualidade da água do corpo hídrico, derivada de imagens de satélite, conforme os padrões de qualidade da água estabelecidos por lei. De acordo com Chen, Zhang e Hallikainen 
(2007), a conexão indica mudança da qualidade da água, especialmente para nitrogênio e fósforo. Os resultados de monitoramento da qualidade da água usando SR mostram a situação atual do ambiente aquático, que pode ser comparado com medições futuras e possíveis problemas na superfície da água. Com essa comparação, pode-se identificar que condições ou indicadores de qualidade da água podem alcançar ou exceder valores críticos estabelecidos na política de recursos hídricos.

O quinto passo aborda a avaliação do monitoramento, que é importante para determinar como o monitoramento da qualidade da água por SR contribui para a política de recursos hídricos na DQA. Nesse sentido, Chen, Zhang e Hallikainen (2007) incluem as seguintes avaliações:

- assegurar que as descobertas são plausíveis e de uso potencial quanto aos padrões de valores críticos de qualidade da água;

- refinar a análise usando diferentes medidas de disponibilidade e ajustar os indicadores ou limiares de segurança;

- avaliar a eficácia do monitoramento da qualidade da água usando SR como suporte para a política de recursos hídricos.

Observa-se que o primeiro caso está relacionado à análise e à validação dos modelos de qualidade da água por satélite, enquanto no segundo verifica-se uma preocupação com a precisão da informação gerada. Já no terceiro caso observa-se uma atenção ao monitoramento, levando em consideração o viés temporal e espacial, proporcionado pelo SR. Logo, as resoluções temporal e espacial devem ser consideradas em uma gestão da qualidade da água por satélite. Conforme Lee (2009), sistemas de SR com alta resolução espacial ( $\sim 30 \mathrm{~m}$ ou melhor) são necessários para o monitorar águas costeiras e continentais. Esses sistemas têm sensores embarcados com canais espectrais que possuem largo comprimento de banda ( $50 \mathrm{~nm}$ ou maior), satisfazendo, assim, os requisitos de sinal-ruído.

Essas características podem afetar o limiar estabelecido em padrões de qualidade da água, sendo necessário realizar avaliações de forma a criar uma amplitude de alerta quanto às informações geradas relacionadas ao próprio limiar da diretiva e ao limiar de precisão do modelo.

\section{SISTEMA DE INFORMAÇÃO GEOGRÁFICA E SENSORIAMENTO REMOTO E SUAS RELAÇÕES COM GESTÃO DE CORPOS HÍDRICOS}

A combinação de SR como fonte de informação e SIG como uma ferramenta analítica permite aos gerenciadores capturar e atualizar todas as informações relevantes (por exemplo, parâmetros de qualidade da água), elaborar planos, simular/implementar, comparar, visualizar e avaliar os resultados a partir da simulação de vários cenários de gestão.

O SIG pode ser usado na avaliação de impacto ambiental em ecossistemas aquáticos e particularmente na avaliação de efeitos cumulativos, que tipicamente requerem a análise de grandes grupos de dados complexos envolvendo ações múltiplas, recursos ambientais e seus indicadores, bem como fatores de causa e efeito associados com a distribuição espacial e temporal (ATKINSON; CANTER, 2011). Nesse sentido, o SIG torna-se uma ferramenta útil devido à sua habilidade de trabalhar com dados geográficos georreferenciados e, por conseguinte, indicado a aplicações espaciais dessa natureza e complexidade (WARNER; DIAB, 2002).

Conforme Atkinson e Canter (2011), camadas de dados (feições vetoriais e imagens) são frequentemente usados para exibição de informações ambientais passadas e atuais. Tais planos de informação podem ser combinados ou eliminados para uma análise específica ou visualização. Além disso, áreas com restrições ambientais, como as relacionadas pelos indicadores selecionados, podem ser facilmente identificadas. Özyavuz (2011) utilizou SIG e SR em diferentes datas para identificar mudanças ocorridas em lago, verificando aumento de áreas residenciais com consequentes descargas no entorno, causando poluição e efeitos negativos na qualidade da água. O Quadro 1 indica como o SIG pode ser usado em análise ambiental. O Quadro 2 mostra as situações de uso e estudo em que pode ser aplicado o SIG.

Atkinson e Canter (2011) ressaltam que o SIG pode ser uma ferramenta valiosa na avaliação atual de condições de ecossistema aquático resultantes do passado e de ações atuais. Tais avaliações podem ser baseadas no desenvolvimento de relações empíricas entre perdas de recursos naturais e degradação ambiental. Özyavuz (2011) mostrou como esse estudo pode ser desenvolvido em ecossistema aquático. O trabalho envolve uso de imagem de satélite, dados de campo e correlações estatísticas, em um ambiente de SIG, utilizando técnicas de geoprocessamento para relacionar as mudanças do ecossistema e o impacto na qualidade da água.

Áreas agrícolas possuem grande potencial na poluição de corpos hídricos, principalmente pelas águas de escoamento que contêm sedimentos de solos erodidos, fertilizantes e pesticidas, visto que podem ser a maior contribuição para a entrada de fontes cumulativas de poluição difusa no local, nas superfícies aquáticas a jusante e a montante. As entradas ocorrem em função de numerosas variáveis, incluindo declividade, classes de solo e tipo de cobertura, intensidade de chuva e taxa de aplicação de químicos (ATKINSON \& CANTER, 2011). Logo, o impacto na qualidade da água de novos projetos agrícolas e as modificações nos existentes podem ser preditos por meio de modelagem. Nesse sentido, a eficácia de combinações de melhores práticas de gestão na redução de entradas de fontes difusas pode, também, ser determinante.

As faixas de comprimentos de onda mais utilizadas para estudos em reservatórios são: do visível $(0,4-0,7 \mu \mathrm{m})$, na qual se tem radiância transmitida (água pura); do infravermelho próximo e médio $(0,7-2,0 \mu \mathrm{m})$, no qual a maior parte da radiância é absorvida, sendo essa faixa usada 
para delineamento do contorno dessa feição, também utilizada para análise do estado trófico e de material em suspensão na água; e, finalmente, do termal (10,4-12,5 $\mu \mathrm{m})$. O Quadro 3 apresenta um resumo das bandas e suas características.

Os fatores que afetam a variabilidade na reflectância de um reservatório ou lago são usualmente determinados por sua configuração física, pela qualidade da água e pelo clima. Os três principais fatores são: profundidade da água, material suspenso (por exemplo, sedimentos inorgânicos, tanino e clorofila) e rugosidade da superfície (LILLESAND \& KIEFER, 1994).

A interação entre a energia eletromagnética, a água e o material contido nela resulta em vários processos que dependem do comprimento

\begin{tabular}{|c|c|}
\hline Fase & Possivel uso do sistema de informações geográficas \\
\hline $\begin{array}{l}\text { Triagem e } \\
\text { escopo }\end{array}$ & $\begin{array}{c}\text { Útil na captura de dados, modelagem espacial, } \\
\text { cálculo na magnitude do impacto e avaliação } \\
\text { de impacto }\end{array}$ \\
\hline $\begin{array}{l}\text { Descrição do } \\
\text { projeto }\end{array}$ & Relações do projeto no contexto geográfico \\
\hline $\begin{array}{l}\text { Descrição das } \\
\text { condições de } \\
\text { base }\end{array}$ & $\begin{array}{l}\text { Documentação e exibição de inventários } \\
\text { biofísicos (por exemplo: vegetação, habitat, uso } \\
\text { do solo, características da superfície da água } \\
\text { etc.), hidrologia, solo, mapas de propriedades, } \\
\text { topografia, rodovia e outros }\end{array}$ \\
\hline $\begin{array}{l}\text { Identificação } \\
\text { do impacto }\end{array}$ & $\begin{array}{l}\text { Uso de análise de camadas para mostrar a } \\
\text { distribuição de poluentes por meio de mapas, ou } \\
\text { integrar os resultados de modelagem da qualidade } \\
\text { da água e análise de sustentabilidade do habitat }\end{array}$ \\
\hline $\begin{array}{l}\text { Predição da } \\
\text { magnitude do } \\
\text { impacto }\end{array}$ & $\begin{array}{l}\text { Usado para a avaliação quantitativa de parte do } \\
\text { recurso afetado por poluentes. Também pode criar } \\
\text { mapas de magnitude do impacto derivado dos } \\
\text { resultados de riscos e da modelagem da qualidade } \\
\text { da água com outros dados em camadas, tal como } \\
\text { susceptibilidade dos solos à erosão ou à salinização } \\
\text { e a magnitude do impacto no corpo hídrico }\end{array}$ \\
\hline $\begin{array}{l}\text { Avaliação da } \\
\text { significância do } \\
\text { impacto }\end{array}$ & $\begin{array}{l}\text { Útil para a exibição da significância do impacto } \\
\text { e como essa variação muda com diferentes } \\
\text { alternativas, incluindo a opção "testemunha" }\end{array}$ \\
\hline $\begin{array}{l}\text { Controle e } \\
\text { mitigação do } \\
\text { impacto }\end{array}$ & $\begin{array}{c}\text { Pode ser usado para identificar áreas onde medidas } \\
\text { de mitigação deveriam ser aplicadas. O SIG também } \\
\text { pode ser utilizado para mostrar temporalmente a } \\
\text { localização geográfica e a extensão das atividades } \\
\text { de mitigação }\end{array}$ \\
\hline $\begin{array}{l}\text { Participação e } \\
\text { consulta pública }\end{array}$ & $\begin{array}{c}\text { O SIG pode ser usado para preparação do material } \\
\text { de apresentação, para explicar o projeto ao público } \\
\text { e para permitir uma resposta rápida a questões e } \\
\text { mudanças sugeridas }\end{array}$ \\
\hline $\begin{array}{l}\text { Monitoramento } \\
\text { e auditoria }\end{array}$ & $\begin{array}{c}\text { Pode-se usar o sistema de informações } \\
\text { geográficas para projetar o programa de } \\
\text { monitoramento, para processar e armazenar } \\
\text { dados de monitoramento, para comparar cenários } \\
\text { atuais com cenários preditos e para apresentação } \\
\text { de dados mostrando a variação local de } \\
\text { poluentes no tempo }\end{array}$ \\
\hline
\end{tabular}

Fonte: adaptado de Atkinson e Canter (2011). de onda e da estrutura atômica da água e seu conteúdo. As relações de balanço de energia são expressas por meio da Equação 1:

$\mathrm{E}_{\mathrm{I}}(\lambda)=\mathrm{E}_{\mathrm{R}}(\lambda)+\mathrm{E}_{\mathrm{A}}(\lambda)+\mathrm{E}_{\mathrm{T}}(\lambda)$

Em que:

a energia incidente $E_{I}(\lambda)$ é em função da energia refletida $E_{R}(\lambda)$, absorvida $\mathrm{E}_{\mathrm{A}}(\lambda)$ e transmitida $\mathrm{E}_{\mathrm{T}}(\lambda)$.

Nessa equação, todos os componentes da energia estão em função do comprimento de onda, o qual tem duas implicações importantes para estudos de reservatórios e lagos. Primeiramente, a proporção da energia

Quadro 2 - Exemplos de estudos que são conduzidos com o uso do sistema de informações geográficas.

Os dados necessários são usados além de um processo estático de avaliação do impacto

Ambientes complexos necessitam ser conhecidos e podem ser usados para mostrar inter-relações do sistema

Apresentação de informações básicas do meio ambiente

Identificação e avaliação de impacto (por exemplo, agropecuária e recursos hídricos)

Quando há disponibilidade de hardwares e softwares

Quando a informação ambiental não é estática

Na análise de cenários

Quando há necessidade de gerar dados para diferentes cenários

Quando há necessidade de interligar atributos espaciais do meio receptor às mudanças no espaço ocasionadas pelo corpo emissor

Fonte: adaptado de Atkinson e Canter (2011).

Quadro 3 - Comprimentos de onda registrados pelo sensor TM (mapeador temático).

\begin{tabular}{|c|c|c|c|}
\hline $\begin{array}{l}\mathrm{N}^{\circ} \mathrm{da} \\
\text { banda }\end{array}$ & Nome da banda & $\begin{array}{l}\text { Comprimento } \\
\text { da banda }(\mu \mathrm{m})\end{array}$ & Características \\
\hline TM 1 & Azul/verde & $0,45-0,52$ & $\begin{array}{l}\text { Boa penetração na } \\
\text { água, forte absorção } \\
\text { pela vegetação }\end{array}$ \\
\hline TM 2 & Verde & $0,52-0,60$ & $\begin{array}{l}\text { Forte reflectância } \\
\text { pela vegetação }\end{array}$ \\
\hline TM 3 & Vermelho & $0,63-0,69$ & $\begin{array}{c}\text { Extrema absorção } \\
\text { da vegetação }\end{array}$ \\
\hline TM 4 & $\begin{array}{l}\text { Infravermelho } \\
\text { próximo }\end{array}$ & $0,76-0,90$ & $\begin{array}{c}\text { Alto contraste solo-água, } \\
\text { extrema reflectância } \\
\text { da vegetação }\end{array}$ \\
\hline TM 5 & $\begin{array}{l}\text { Infravermelho } \\
\text { médio próximo }\end{array}$ & $1,55-1,75$ & $\begin{array}{l}\text { Sensibilidade forte } \\
\text { a umidade }\end{array}$ \\
\hline TM 6 & $\begin{array}{l}\text { Infravermelho } \\
\text { termal }\end{array}$ & $10,4-12,5$ & $\begin{array}{l}\text { Sensibilidade forte } \\
\text { à umidade do solo } \\
\text { e da vegetação }\end{array}$ \\
\hline TM 7 & $\begin{array}{l}\text { Infravermelho } \\
\text { médio }\end{array}$ & $2,08-2,35$ & $\begin{array}{l}\text { Boa discriminação } \\
\text { geológica }\end{array}$ \\
\hline
\end{tabular}


refletida, absorvida e transmitida irá variar para diferentes corpos hídricos, dependendo da sua condição ambiental e ecológica. Em um segundo momento, para uma condição particular do reservatório/lago (por exemplo, profundidade da água), a proporção de energia refletida, absorvida e transmitida irá variar em diferentes comprimentos de onda. Assim, duas feições (águas rasas e profundas) podem ser distinguíveis em uma banda espectral e ser muito diferentes em outra banda. Dessa forma, cada reservatório e sua área de contribuição podem prover características distintas de assinaturas espectrais para uma determinada análise.

O uso do SR na gestão de reservatórios é baseado no fato de que as consequências de eutrofização e o aumento da produtividade estão associados com a mudança nas propriedades ópticas da massa hídrica. Aumento da clorofila-a está associado com diminuição na quantidade relativa de energia da banda azul $(0,45-0,52 \mu \mathrm{m})$ e aumento na verde $(0,52-0,60 \mu \mathrm{m})$ (FRIESE et al., 2010), e geralmente se utiliza para sua determinação a razão entre os canais 704 e 675 nm (LI et al., 2010). Aumento de sólidos suspensos está associado com aumento na energia refletida, e o pico de reflectância moverá na direção de comprimentos de onda maiores (LILLESAND \& KIEFER, 1994). Essas mudanças podem ser medidas por técnicas de SR (NELLIS; HARRINGTON; WU, 1998; ONDERKA \& PEKÁROVÁ, 2008; SONG et al., 2010).

Muitas observações da superfície da Terra vistas do espaço são realizadas por instrumentos abordo de satélites, os quais têm numerosos sensores operando em diferentes comprimentos de onda. Segundo Baban (1999), os instrumentos mais utilizados, como o Mapeador Temático Landsat ${ }^{\mathrm{T \omega}}$, o sistema para observação da Terra (SPOT) e o avançado radiômetro de alta resolução (AVHRR), têm grupos de sensores que operam na maior parte dos comprimentos de onda relevantes para estudos de reservatórios e lagos. Isso provê uma estrutura básica para limnologistas, hidrologistas e ecologistas, já que suas coberturas espectrais correspondem a regiões com alta interação da energia, tanto do sol (incidente) quanto da Terra (refletida/emitida), e possuem janelas atmosféricas, em que a energia eletromagnética tem menores distorções.

Segundo Baban (1999), dados orbitais podem ser usados na gestão de corpos hídricos, porém nunca poderão substituir redes de observação hidrolimnológica convencional em reservatórios ou lagos.

O SR e o SIG têm extraído e gerado, com sucesso, informações relevantes relacionadas às características da bacia hidrográfica, como, por exemplo, mapeamento das mudanças das terras agrícolas em um determinado período (AHMED et al., 2009; HONISCH; HELLMEIER; WEISS, 2002; ZHANG et al., 2009; ZHANG; ZHENGJUN; XIAOXIA, $2009 b$ ), além de providenciar dados de qualidade da água em sistemas aquáticos (reservatório, lagos e rios) para gestão, incluindo: clorofila-a, fósforo total, profundidade secchi, sólidos suspensos, salinidade e temperatura (BURRAGE et al., 2003; GIARDINO et al., 2001; GITELSON; STARK; DOR, 1997; HONISCH; HELLMEIER; WEISS,
2002; HILTON; BUCKLAND; IRONS, 2002; NOVO et al., 2006; REIS et al., 2003; WANG et al., 2010).

\section{ESTRATÉGIA DE GESTÃO DA QUALIDADE DA ÁGUA POR MEIO DE GEOPROCESSAMENTO}

É abordada aqui a elaboração de estratégias de gestão de um corpo hídrico com base em informações espectrais e de campo, tendo como aplicação reservatórios de armazenamento superficial de água e suas áreas de contribuição.

A comunidade europeia vem aplicando geotecnologias associadas, como a DQA, para otimizar as estratégias de gestão dos recursos hídricos. Nesse sentido, será elaborada uma estrutura integrada por SR e SIG aliada à Resolução n 357/2005 (BRASIL, 2005), que dispõe sobre a classificação dos corpos de água e estabelece diretrizes ambientais para o seu enquadramento. Os parâmetros a serem considerados serão aqueles possíveis de serem modelados por SR, como, por exemplo: clorofila-a, cianobactérias, turbidez, profundidade secchi, temperatura da superfície, sólidos suspensos, carbono orgânico dissolvido, nitrato (obtido de forma indireta e mostrado em Montzka et al., 2008, e Hajhamad \& Almasri, 2009), e outros que podem ser correlacionados com a resposta espectral da água. Alguns parâmetros poderão ser modelados e espacializados utilizando correlações estatísticas a partir de outros parâmetros.

A Figura 1 mostra a proposta de integração entre SR, SIG e Resolução no 357/2005 (BRASIL, 2005), além de dados de campo, de forma a gerar dados de saída para a tomada de decisão pelos gestores. Busca-se, dessa forma, uma visão sinótica e holística das interações e mudanças que ocorrem no meio aquático e suas relações com a área de contribuição do objeto geográfico em estudo.

Observa-se, pela Figura 1, que existe coleta tanto limnológica quanto espectral georreferenciada de informação no campo. No caso de não haver dados espectrais de campo, haverá uma simulação por meio do software WASI (water colour simulator), detalhado em Gege (2004). Após a coleta de informação, pode ser feito o mapeamento temático por meio da modelagem em SIG e, posteriormente, uma validação com verdade de campo; caso não esteja dentro do erro aceitável, haverá novamente uma modelagem, com novas informações, para aperfeiçoar o modelo. Uma outra entrada de dados é feita com imagens de satélites adequadas para o objetivo proposto e o corpo hídrico em estudo. Essas imagens são processadas, momento em que são extraídas informações (por exemplo, parâmetros de qualidade da água - PQAs), e validadas de forma a atender a precisão aceitável, caso contrário serão feitos novos levantamentos para atingir o erro aceitável.

Todas as informações geradas e processadas são armazenadas em um banco de dados temático, e a partir desse ponto podem ser gerados índices, como uma variável (clorofila, turbidez, temperatura), o índice 


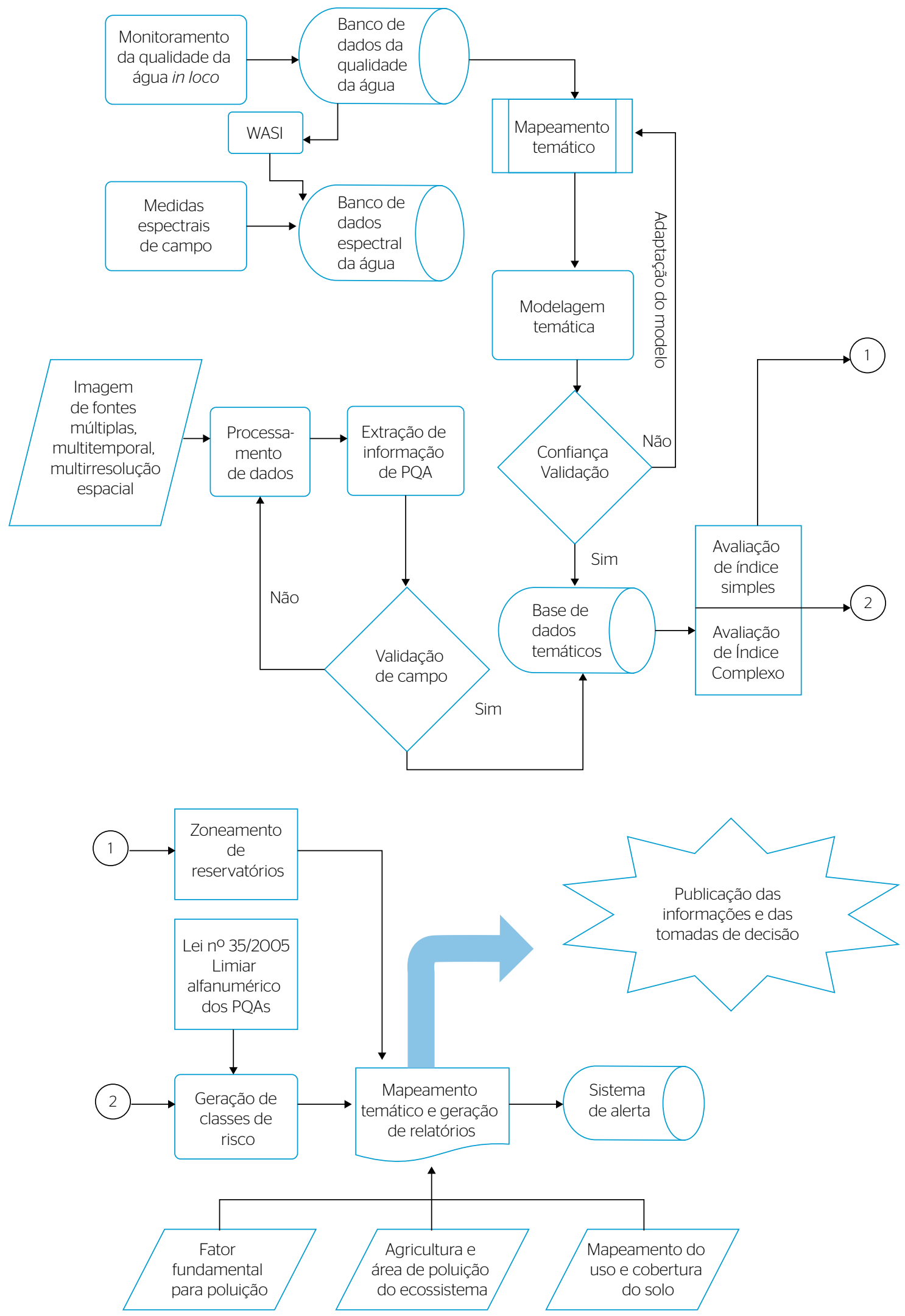

WASI: water colour simulator; PQA: parâmetro de qualidade da água.

Figura 1 - Fluxograma da integração sistema de informações geográficas, sensoriamento remoto e Resolução no 357/2005, no processamento da qualidade da água, para gestão e tomada de decisão em reservatórios de abastecimento. 
de estado trófico (IET), o índice de qualidade da água (IQA), e o índice de conformidade ao enquadramento (AMARO, 2009; ANA, 2012), do Canadian Council of Ministers of the Environment (CCME). Com esses índices também pode ser gerado o zoneamento do reservatório e, consequentemente, o mapeamento temático e o relatório das zonas limnológicas. Essas variáveis e/ou índices podem ser cruzados com informações tabulares descritas na Resolução nº 357/2005 (BRASIL, 2005), considerando os limiares de aceitação de cada parâmetro, de forma a verificar, pixel a pixel, se o corpo hídrico está fora do estabelecido por lei. Após essa comparação, tais informações podem ser classificadas em classes e associadas ao risco, ou seja, quais zonas estão fora do padrão. Pode-se gerar mapeamento temático e relatórios verificando as possíveis fontes de poluição, seja pontual (por exemplo, esgotos) ou difusa (por exemplo, agricultura), de forma a servir de base para um sistema de alerta e, por conseguinte, para a tomada de decisão em uma gestão estratégica e sustentável. Por outro lado, como visto na Figura 1, o SIG proporciona meios de divulgação, seja por e-mail, FTP ou WebGIS, tornando-se uma ferramenta de gestão participativa e transparente, em forma de mapa ou relatórios disponibilizados à comunidade.

\section{CONSIDERAÇÕES FINAIS}

O Brasil possui corpos hídricos, como reservatórios, que contemplam superfícies de dezenas de $\mathrm{km}^{2}$. Dessa forma, observa-se que o monitoramento por técnicas convencionais é muito dispendioso em termos de tempo e custos, como também para comparação entre pontos amostrais. Nesse sentido, o SR aliado a técnicas de geoprocessamento traz vantagens relacionadas a essas duas exigências. Contudo, há necessidade de modelagem de algoritmos para cada corpo hídrico, principalmente pelas peculiaridades geográficas existentes em sua área de influência, ressaltando o erro existente em cada modelo. Nesse sentido, o SR não vem substituir o monitoramento de campo, mas sim fazer parte do sistema de monitoramento integrado de um determinado corpo hídrico.

Outro ponto fundamental é a necessidade de estudo de parâmetros de qualidade de água em reservatórios por meio de SR interligado aos limiares estabelecidos na Resolução no 357/2005 (BRASIL, 2005). É necessário realizar estudos com bases estatísticas visando identificar a precisão dos modelos de determinação de parâmetros de qualidade de água relacionados aos limites aceitáveis para cada um. Verifica-se que existem estudos em andamento na Europa, principalmente com relação à clorofila-a, objetivando o seu mapeamento para relacionar com os padrões da DQA. Assim, fica visível o importante papel das geotecnologias na política de recursos hídricos, notadamente no monitoramento e na geração de informações fundamentais à tomada de decisão dos gestores.

Este trabalho mostra uma proposta de integração entre quatro pilares: SR, SIG, Resolução no 357/2005 (BRASIL, 2005) e dados de campo. Com isso, busca-se fornecer dados de saída para a tomada de decisão de gestores. Também é apresentada uma visão sinótica e holística das interações e mudanças do meio aquático e suas relações com a área de estudo.

\section{REFERÊNCIAS}

AHMED, M.H.; EL LEITHY, B.M.; THOMPSON, J.R.; FLOWER, R.J.; RAMDANI, M.; AYACHE, F.; HASSAN, S.M. (2009) Application of remote sensing to site characterisation and environmental change analysis of North African coastal lagoons. Hydrobiologia, v. 622, p. 147-171.

ALCÂNTARA, E.H.; NOVO, E.M.L.M.; STECH, J.L. (2011) Novas tecnologias para o monitoramento e estudo de reservatórios hidrelétricos e grandes lagos. Rio de Janeiro: Parênteses. 328p.

ALEXANDRIDIS, T.K.; TAKAVAKOGLOU, V.; CRISMAN, T.L.; ZALIDIS, G.C. (2007) Remote sensing and GIS techniques for selecting a sustainable scenario for Lake Koronia, Greece. Environmental Management, v. 39, p. n. 2, 278-290.

AMARO, C.A. (2009) Proposta de um índice para avaliação de conformidadeda qualidadedos corpos hídricosao enquadramento. Dissertação (Mestrado). Departamento de Engenharia Hidráulica e Sanitária, Escola Politécnica da Universidade de São Paulo.

ANA - Agência Nacional de Águas. (2012) Panorama da qualidade das águas superficiais do Brasil: 2012. Agência Nacional de Águas. Brasília: ANA.
ANDERSON, M.C.; NORMAN, J.M.; KUSTAS, W.P.; HOUBORG, R. STARKS, P.J.; AGAM, N. (2008) A thermal-based remote sensing technique for routine mapping of land-surface carbon, water and energy fluxes from field to regional scales. Remote Sensing of Environment, v. 112, n. 12, p. 4227-4241.

ARTIGAS, F.; MARTI, A.; YAO, N.; PECHMANN, I. (2008) Chlorophyll detection and mapping of shallow water impoundments using image spectrometry. Research Letters in Ecology, p. 1-4.

ATKINSON, S.F. \& CANTER, L.W. (2011). Assessing the cumulative effects of projects using geographic information systems. Environmental Impact Assessment Review, v. 31, n. 5, p. $457-464$

BABAN, S.M.J. (1999). Use of remote sensing and geographical information systems in developing lake management strategies. Hydrobiologia, v. 395/396, p. 211-226.

BAKER, D.; YOUNG, J.; AROCENA, J.M. (2000). An integrated approach to reservoir management: the Williston reservoir case study. Environmental Management, v. 25, n. 5, p. 565-578. 
BERGAMINO, N.; HORION, S.; STENUITE, S.; CORNET, Y.; LOISELLE, S.; PLISNIER, P-D.; DESCY, J-P. (2010) Spatio-temporal dynamics of phytoplankton and primary production in Lake Tanganyika using a MODIS based bio-optical time series. Remote Sensing of Environment, v. 114, n. 4, p. 772-780.

BRASIL. (2005) Resolução CONAMA no 357/2005, de 17 de março de 2005. Dispõe sobre a classificação dos corpos de água e diretrizes ambientais para o seu enquadramento, bem como estabelece as condições e padrões de lançamento de efluentes, e dá outras providências. Oficial da União, 18 de março de 2005.

BURRAGE, D.M.; HERON, M.L.; HACKER, J.M.; MILLER, J.L.; STIEGLITZ, T.C.; STEINBERG, C.R.; PRYTZ, A. (2003) Structure and influence of tropical river plumes in the Great Barrier Reef: application and performance of an airborne sea surface salinity mapping system. Remote Sensing of Environment, v. 85, p. 204-220.

CHENG, K.-S. \& LEI, T.-C. (2001) Reservoir trophic state evaluation using landsat TM images. Journal of the American Water Resources Association, v. 37, n. 5, p. 1321-1334.

CHEN, Q.; ZHANG, Y.; HALLIKAINEN, M. (2007) Water quality monitoring using remote sensing in support of the EU water framework directive (WFD): a case study in the Gulf of Finland. Environment Monitoring Assessment, v. 124, p. 157-166.

CHEN, S.; FANG, L.; LI, H.; CHEN, W.; HUANG, W. (2011). Evaluation of a three-band model for estimating chlorophyll-a concentration in tidal reaches of the Pearl River Estuary, China. ISPRS Journal of Photogrammetry and Remote Sensing, v. 66, n. 3, p. 356-364.

DUAN, H.; ZHANG, Y.; ZHANG, B.; SONG, K.; WANG, Z.; LIU, D.; LI, F. (2008) Estimation of chlorophyll-a concentration and trophic states for inland lakes in Northeast China from Landsat TM data and field spectral measurements. International Journal of Remote Sensing, v. 29, n. 3, p. 767-786.

FRIESE, M.; MIGDALL, S.; BACH, H.; KRÄTZ, D.; BORCHARDT, D.; HANK, T.; MAUSER, W. (2010) Water quality assessment using hyperspectral techniques for the river main and its riparian water. In: Hyperspectral 2010 Workshop. Anais... Frascati, Itália. Disponível em: <http://earth.esa.int/workshops/hyperspectral_2010/papers/ s4_1friese.pdf>. Acesso em: 4 nov. 2011.

FUCHS, H.; MAGDON, P.; KLEINN, C.; FLESSA, H. (2009) Estimating aboveground carbon in a catchment of the Siberian forest tundra: Combining satellite imagery and field inventory. Remote Sensing of Environment, v. 113, n. 3, p. 518-531.

FUKUE, M.; SATO, Y.; MULLIGAN, C. Monitoring of surface water quality, sustainable built environment. UNESCO: EOLSS. Disponível em: <http://www.eolss.net/Sample-Chapters/C15/E1-32-07-O2.pdf>. Acesso em: 23 maio 2011.

GEGE, P. (2004) The water color simulator WASI: an integrating software tool for analysis and simulation of optical in situ spectra. Computers \& Geosciences, v. 30, n. 5, p. 523-532.

GIARDINO, C.; PEPE, M.; BRIVIO, P.A.; GHEZZI, P.; ZILIOLI, E. (2OO1) Detecting chlorophyll, Secchi disk depth and surface temperature in a sub-alpine lake using Landsat imagery. The Science of the Total Environment, v. 268, p. 19-29.

GITELSON, A.; STARK, R.; DOR, I. (1997) Quantitative near-surface remote sensing of wastewater quality in oxidation ponds and reservoirs: a case study of the Naan system. Water Environment Research, v. 69, n. 7, p. 1263-1271.

GITELSON, A.A.; DALL'OLMO, G.; MOSES, W.; RUNDQUIST, D.C:; BARROW, T.; FISHER, T.R.; GURLIN, D.; HOLZ, J. (2008) A simple semianalytical model for remote estimation of chlorophyll-a in turbid waters: validation. Remote Sensing of Environment, v. 112, p. 3582-3593.

GODDIJN-MURPHY, L.; DAILLOUX, D.; WHITE, M.; BOWERS, D. (2009) Fundamentals of in situ digital camera methodology for water quality monitoring of coast and ocean. Sensors, v. 9, n. 7, p. 5825-5843.

GREGOR, J. \& MARSÁLEK, B. (2004) Freshwater phytoplankton quantification by chlorophyll a: a comparative study of in vitro, in vivo and in situ methods. Water Research, v. 38, n. 3, p. 517-522.

HAJHAMAD, L. \& ALMASRI, M.N. (2009) Assessment of nitrate contamination of groundwater using lumped-parameter models. Environmental Modelling and Software, v. 24, p. 1073-1087. doi: 10.1016/j.envsoft.2009.02.014

HADJIMITSIS, D.G. \& CLAYTON, C. (2O11) Field spectroscopy for assisting water quality monitoring and assessment in water treatment reservoirs using atmospheric corrected satellite remotely sensed imagery. Remote Sensing, v. 3, n. 2, p. 362-377.

HILTON, J.; BUCKLAND, P.; IRONS, G.P. (2002) An assessment of a simple method for estimating the relative contributions of point and diffuse source phosphorus to in-river phosphorus loads. Hydrobiologia, v. 472, p. 77-83

HONISCH, M.; HELLMEIER, C.; WEISS, K. (2OO2) Response of surface and subsurface water quality to land use changes. Geoderma, v. 105, p. $277-298$.

JESSEL, B. \& JACOBS, J. (2005) Land use scenario development and stakeholder involvement as tools for watershed management within the Havel River Basin. Limnologica, v. 35, n. 3, p. 220-233.

JOHNSON, L.E. (2009) Geographic information systems in water resources engineering. Boca Raton, Florida: CRC Press, Taylor \& Francis Group. 340p.

KAY, J.E.; KAMPF, S.K.; HANDCOCK, R.N.; CHERKAUER, K.A.; GILLESPIE, A.R.; BURGES, S.J. (2OO5) Accuracy of lake and stream temperatures estimated from thermal infrared images. Journal of the American Water Resources Association, v. 41, n. 5, p. 1161-1175.

KLOIBER, S.M.; BREZONIK, P.L.; BAUER, M.E. (2002) Application of Landsat imagery to regional-scale assessments of lake clarity. Water Research, v. 36, p. 4330-4340.

LE, C.F.; LI, Y.M.; ZHA, Y.; SUN, D.Y.; HUANG, C.C.; LU, H. (2009) A fourband semi-analytical model for estimating chlorophyll-a in highly turbid lakes: the case of Taihu Lake, China. Remote Sensing of Environment, v. 113, n. 6, p. 1175-1182. 
LEE, Z.P. (2009) Applying narrowband remote-sensing reflectance models to wideband data. Applied Optics, v. 48, n. 17, p. 3177-3183.

LEUVEN, R.S.E.W.; POUDEVIGNE, I.; TEEUW, R.M. (2002) Application of geographical information systems and remote sensing in river studies. Leiden: Backhuys Publishers. 247p.

LI, L.; SENGPIEL, R.E.; PASCUAL, D.L.; TEDESCO, L.P.; WILSON, J.S.; SOYEUX, E. (2010) Using hyperspectral remote sensing to estimate chlorophyll- a and phycocyanin in a mesotrophic reservoir. International Journal of Remote Sensing, v. 31, n. 15 , p. 4147-4162.

LILLESAND, T.M. \& KIEFER, R.W. (1994) Remote sensing and image interpretation. 3. ed. New York: John Wiley \& Sons. 750p.

LOPES, H.L.; CANDEIAS, A.L.B.; ACCIOLY, L.J.O.; SOBRAL, M.C.M.; PACHECO, A.P. (2010) Parâmetros biofísicos na detecção de mudanças na cobertura e uso do solo em bacias hidrográficas. Revista Brasileira de Engenharia Agrícola e Ambiental, v. 14, п. 11, p. 1210-1219.

LOPES, H.; SOBRAL, M.C.; GUNKEL, G.; CANDEIAS, A.L. (2015) Comportamento espacial da clorofila-a no reservatório de Itaparica, rio São Francisco. Engenharia Sanitária e Ambiental, v. 20, n. 3, p. 475-484.

MA, R. \& DAl, J. (2005) Investigation of chlorophyll-a and total suspended matter concentrations using Landsat ETM and field spectral measurement in Taihu Lake, China. International Journal of Remote Sensing, v. 26, n. 13, p. 2779-2795.

MATOS, R.C.M.; CANDEIAS, A.L.B.; AZEVEDO, J.R.G.; HATTERMANN, F.F.; KOCH, H.; KLEINSCHMIT, B. (2015) Análise multitemporal do Albedo, NDVI e temperatura no entorno do Reservatório de Itaparica - PE: anos de 1985 e 2010. Revista Brasileira de Cartografia, v. 67, n. 3, p. 545-554.

MATSUOKA, Y.; KAWAMURA, H.; SAKAIDA, F.; HOSODA K. (2011) Retrieval of high-resolution sea surface temperature data for Sendai Bay, Japan, using the Advanced Spaceborne Thermal Emission and Reflection Radiometer (ASTER). Remote Sensing of Environment, v. 115, n. 1, p. 205-213.

MEERSMANS, J.; DE RIDDER, F.; CANTERS, F.; DE BAETS, S.; VAN MOLLE, M. (2008) A multiple regression approach to assess the spatial distribution of Soil Organic Carbon (SOC) at the regional scale (Flanders, Belgium). Geoderma, v. 143, n. 1, p. 1-13.

MELO, G.L. (2007) Estudo da qualidade da água no reservatório de Itaparica localizado na Bacia do Rio São Francisco. 97p. Dissertação (Mestrado) - Programa de Pós-Graduação em Engenharia Civil, Universidade Federal de Pernambuco, Recife.

MELO, G.L. (2O11) Avaliação da qualidade da água em reservatórios de bacias hidrográficas do nordeste setentrional interligadas com o Rio São Francisco. 144p. Tese (Doutorado) - Programa de Pós-Graduação em Engenharia Civil, Universidade Federal de Pernambuco, Recife.

MIRANDA, J.I. (2010) Fundamentos de Sistemas de Informações Geográficas. 2. ed. Brasília: Embrapa Informática Tecnológica. 425p.
NELLIS, M.D.; HARRINGTON JR., J.A.; WU, J. (1998) Remote sensing of temporal and spatial variations in pool size, suspended sediment, turbidity, and Secchi depth in Tuttle Creek Reservoir, Kansas: 1993. Geomorphology, v. 21, n. 3, p. 281-293.

MONTZKA, C.; CANTY, M.; KREINS, P.; KUNKEL, R.; MENZ, G.; VEREECKEN, H.; WENDLAND, F. (2008) Multispectral remotely sensed data in modelling the annual variability of nitrate concentrations in the leachate. Environmental Modelling \& Software, v. 23, n. 8, p. 1070-1081

NOVO, E.M.L.M.; BARBOSA, C.C.F.; FREITAS, R.M.; SHIMABUKURO, Y.E.; MELACK, J.M.; PEREIRA FILHO, W. (2006) Seasonal changes in chlorophyll distributions in Amazon floodplain lakes derived from MODIS images. Limnology, v. 7, n. 3, p. 153-161.

OLIVEIRA, L.M.M.; MONTENEGRO, S.M.G.L.; SILVA, B.B; ANTONINO, A.C.D.; MOURA, A.E.S.S. (2014) Evapotranspiração real em bacia hidrográfica do Nordeste brasileiro por meio do SEBAL e produtos MODIS. Revista Brasileira de Engenharia Agrícola e Ambiental, v. 18, n. 10, p. $1039-1046$

ONDERKA, M. \& PEKÁROVÁ, P. (2008) Retrieval of suspended particulate matter concentrations in the Danube River from Landsat ETM data. Science of the Total Environment, v. 397, p. 238-243.

ÖZYAVUZ, M. (2011) Determination of temporal changes in lakes Mert and Erikli using remote sensing and Geographic Information Systems. Journal of Coastal Research, v. 27, n. 1, p. 174-181.

PAES, R.F.C.; CANDEIAS, A.L.B.; SOBRAL, M.C.M. (2010) Sistemas de informações geográficas para subsidiar a tomada de decisão na gestão ambiental de reservatórios. In: III Simpósio Brasileiro de Ciências Geodésicas e Tecnologias da Geoinformação, Recife. Anais... Recife.

POWELL, S.L.; COHEN, W.B.; HEALEY, S.P.; KENNEDY, R.E.; MOISEN, G.G.; PIERCE, K.B.; OHMANN, J.L. (2010) Quantification of live aboveground forest biomass dynamics with Landsat time-series and field inventory data: a comparison of empirical modeling approaches. Remote Sensing of Environment, v. 114, p. 1053-1068.

REIS, R.S.; NOVO, E.M.L.M.; ESPÍNDOLA, E.L.G.; SEVERI, W. (2003) Uso de imagens Landsat e dados de campo para avaliar a distribuição espacial de material em suspensão em reservatórios do semi-árido brasileiro. In: Simpósio Brasileiro de Sensoriamento Remoto (SBSR), 11., 2003, Belo Horizonte. Anais... Belo Horizonte: INPE. p. 2569-2577. CD-ROM.

RODRIGUES, J.O;; ANDRADE, E.M.; TEIXEIRA, A.S.; SILVA, B.B. (2009) Sazonalidade de variáveis biofísicas em regiões semiáridas pelo emprego do sensoriamento remoto. Engenharia Agrícola, Jaboticabal, v. 29, n. 3, p. 452-465.

SÁNCHEZ, L.E. (2008) Avaliação de impacto ambiental: conceitos e métodos. São Paulo: Oficina de Textos. 445p.

SASS, G.Z.; CREED, I.F.; BAYLEY, S.E.; DEVITO, K. J. (2007) Understanding variation in trophic status of lakes on the Boreal Plain: A 20 year retrospective using Landsat TM imagery. Remote Sensing of Environment, v. 109, p. 127-141. 
SILVEIRA, C.S. \& SILVA, V.V. (2010) Dinâmicas de regeneração, degeneração e desmatamento da vegetação provocadas por fatores climáticos e geomorfológicos: uma análise geoecológica através de SIG. Revista Árvore, v. 34, n. 6, p. 1025-1034.

SOBRAL, M.C.M.; GUNKEL, G.; BARROS, A.M.L.; PAES, R.; FIGUEIREDO, R.C. (2008) Classificação de corpos d'água segundo a Diretiva-Quadro da Água da União Européia - 2000/60/CE. Revista Brasileira de Ciências Ambientais, n. 11, p. 30-39.

SONG, K:; DONGMEI, L;; LIU, D; WANG, Z; LI, L.; ZHANG, B; WANG, Y. (2010) Retrival of total suspended matter (TSM) using remotely sensed images in Shitoukoumen Reservoir, northeast China. IEEE, p. 405-408.

TRIPATHI, N.K.; VENKOBACHAR, C.; SINGH, R.K.; SINGH, S.P. (1998) Monitoring the pollution of river Ganga by tanneries using the multiband ground truth radiometer. ISPRS Journal of Photogrammetry \& Remote Sensing, v. 53, n. 4, p. 204-216.

TUCCl, C.E.M. \& MENDES, C.A. (2006) Avaliação ambiental integrada de bacia hidrográfica. Brasília: MMA. 302p.

XINGTANG,H.; BING,Z:; XIA,Z:; QINGXI,T.:LANFEN,Z:; QIAO,W:; JANLIN, $Y$. (2004) A new architecture for remote sensing environmental monitoring system REMS: design and implementation. IEEE Geoscience and Remote Sensing, v. 3, p. 2015-2018.

WANG, F.; HAN, L.; KUNG, H.T.; VAN ARSDALE, R.B. (2006) Applications of Landsat-5 TM imagery in assessing and mapping water quality in Reelfoot Lake, Tennessee. International Journal of Remote Sensing, v. 27, n. 23, p. 5269-5283.

WANG, H.; HLADIK, C.M.; HUANG, W.; MILLA, K.; EDMISTON, L.; HARWELL, M.A.; SCHALLES, J.F. (2010) Detecting the spatial and temporal variability of chlorophyll-a concentration and total suspended solids in Apalachicola Bay, Florida using MODIS imagery. International Journal of Remote Sensing, v. 31, n. 2, p. 439-453.
WANG, M.; SHI, W.; TANG, J. (2011) Water property monitoring and assessment for China's inland Lake Taihu from MODISAqua measurements. Remote Sensing of Environment, v. 115, n. 3, p. 841-854.

WARNER, L.L. \& DIAB, R.D. (2002) Use of geographic information systems in an environmental impact assessment of an overhead power line. Impact Assessment of Project Appraisal, v. 20, n. 1, p. 39-47.

ZALIDIS, G.; S. STAMATIADIS; V. TAKAVAKOGLOU; K. ESKRIDGE; N. MISOPOLINOS. (2002) Impacts of agricultural practices on soil and water quality in Mediterranean region and proposed assessment methodology. Agriculture, Ecosystems and Environment, v. 88, n. 2, p. 137-146.

ZHANG, J.; ZHENGJUN, L.; XIAOXIA, S. (2009) Changing landscape in the Three Gorges Reservoir Area of Yangtze River from 1977 to 2005: land use/land cover, vegetation cover changes estimated using multi-source satellite data. International Journal of Applied Earth Observation and Geoinformation, v. 11, n. 6, p. 403-412.

ZHANG, Y.; LIN, S.; QIAN, X.; WANG, Q.; QIAN, Y.; LIU, J.; GE, Y. (2O11) Temporal and spatial variability of chlorophyll a concentration in Lake Taihu using MODIS time-series data. Hydrobiologia, v. 661, n. 1, p. 235-250.

ZHANG, Y.; WANG, Y.; WANG, Y.; XI, H. (2009). Investigating the impacts of Landuse-landcover (LULC) change in the Pearl River delta region on water quality in the Pearl River estuary and Hong Kong's Coast. Remote Sensing, v. 1, n. 4, p. 1055-1064.

ZHENGJUN, W.; JIANMING, H.; GUISEN, D. (2008) Use of satellite imagery to assess the trophic state of Miyun Reservoir, Beijing, China. Environmental Pollution, v. 155, n. 1, p. 13-19. 